\title{
O sistema público de saúde e as ações de reabilitação no Brasil
}

\author{
Carla Trevisan Martins Ribeiro, ${ }^{1}$ Marcia Gonçalves Ribeiro, ${ }^{2}$ \\ Alexandra Prufer Araújo, ${ }^{2}$ Lívia Rodrigues Mello, ${ }^{3}$ \\ Luciana da Cruz Rubim ${ }^{3}$ e Joyce Espírito Santo Ferreira ${ }^{3}$
}

Como citar Ribeiro CTM, Ribeiro MG, Araújo AP, Mello LR, Rubim LC, Ferreira JES. O sistema público de saúde e as ações de reabilitação no Brasil. Rev Panam Salud Publica. 2010;28(1):43-8.

RESUMO Objetivo. Realizar um levantamento histórico das ações de reabilitação no contexto do Sistema Único de Saúde (SUS).

Métodos. Pesquisa de materiais publicados entre 1980 e 2009 nas bases de dados SciELO, LILACS e MEDLINE. Foram utilizadas as seguintes palavras-chave em português: Sistema Único de Saúde, reabilitação, políticas de saúde, assistência médica, história. Em inglês foram utilizadas as palavras rehabilitation $e$ public health. Também foram pesquisadas as leis federais e os manuais do Ministério da Saúde, junto à Coordenação de Programas de Reabilitação da Cidade do Rio de Janeiro, na biblioteca da Fundação Instituto Oswaldo Cruz e na base de dados BIREME.

Resultados. Obteve-se apenas um pequeno número de publicações (quatro livoros, três manuais do Ministério da Saúde, quatro artigos nacionais, uma dissertação de mestrado e uma tese de doutorado). Entretanto, a análise desses materiais mostrou que, como muitos municípios ainda não são capazes de garantir o direito universal e integral à saúde, as ações de reabilitação são muitas vezes levadas a cabo de forma precária, sem apoio de uma política adequada e integral. Por outro lado, houve avanços concretos no sentido de ampliar o atendimento à população com necessidades especiais.

Conclusões. Ainda persistem fatores que dificultam o alcance de ótimos resultados na atenção à pessoa com necessidades especiais. Cabe especialmente aos municípios o desafio de assumir o planejamento das ações, estabelecendo a oferta adequada de serviços e promovendo, assim, a equidade de acesso e a integralidade da assistência.

Palavras-chave Políticas públicas; reabilitação; Sistema Único de Saúde; Brasil.

O conceito de reabilitar inclui diagnóstico, intervenção precoce, uso adequado de recursos tecnológicos, continuidade de atenção e diversidade de modalidades de atendimentos visando à compensação da perda da funcionalidade do indivíduo, à melhoria ou manutenção da

1 Universidade Federal do Rio de Janeiro (UFRJ), Programa de Pós-Graduação em Clínica Médica. Correspondência: carlatrevisan@ig.com.br

2 UFRJ, Departamento de Pediatria, Rio de Janeiro (RJ), Brasil.

3 UFRJ, Programa de Graduação em Fisioterapia, Rio de Janeiro (RJ), Brasil. qualidade de vida e à inclusão social (1, 2). Como afirmam Coelho e Lobo (1), o trabalho de reabilitação deve englobar tanto o aspecto técnico quanto o aspecto da cidadania do indivíduo com deficiência, que tem o direito de fazer escolhas e de ser o autor de sua própria história.

No Brasil, de acordo com a Constituição de 1988, a saúde é um direito de todo o cidadão e deve ser garantida pelo Estado (3), sendo o direito à habilitação e à reabilitação das pessoas portadoras de deficiência garantido por lei federal (4).
Contudo, esse tipo de prerrogativa é bastante recente na história do país.

A saúde pública no Brasil tem duas fases bastante distintas, sendo a criação do Sistema Único de Saúde (SUS) o marco divisor dessas fases (3). Antes da reforma sanitária e da criação do SUS, em 1988, a saúde era restrita a uma parcela da população que contribuía com um seguro social para desfrutar de tal benefício. As ações de saúde pública eram de caráter preventivo e coletivo, com escassas exceções de assistência à saúde voltada para 
doenças específicas ou grupos populacionais $(3,5)$. Nessa época, a assistência ao portador de deficiência e ao doente mental baseava-se na caridade e na filantropia $(1,2)$. A reabilitação não ficava a cargo de nenhuma esfera governamental, com exceção dos centros de reabilitação profissional do Instituto Nacional de Previdência Social (INPS) $(2,6)$.

Após a instituição do SUS, a saúde passou a ser um direito de todos e um dever do Estado. A partir de então houve uma descentralização da política de saúde e os estados e municípios passaram a administrar serviços comprometidos com os princípios da proposta de reforma sanitária a fim de garantir o direito universal e integral à saúde $(3,7)$. Entretanto, os programas de reabilitação brasileiros passaram a ter administração federal e consequente organização centralizada. O governo federal passou a desenvolver programas de atendimento em reabilitação para deficientes na rede pública de saúde (2).

Entender o impacto das transformações das políticas de saúde no Brasil, tendo como eixo principal a análise do direito à saúde e da reabilitação de pessoas portadoras de deficiência, é essencial para a compreensão crítica do que significa atualmente o sistema de saúde para essa população. Contudo, são escassos os estudos sobre programas e políticas de reabilitação no Brasil.

Dessa forma, o objetivo deste trabalho é apresentar uma revisão histórica da literatura sobre políticas de saúde pública no Brasil quanto ao surgimento das ações de reabilitação para portadores de necessidades especiais.

\section{MATERIAIS E MÉTODOS}

Um levantamento bibliográfico foi realizado nas bases de dados SciELO, LILACS e MEDLINE para o período de 1980 a 2009. Foram utilizadas as seguintes palavras-chave em português: Sistema Único de Saúde, reabilitação, políticas de saúde, assistência médica, história. Foram combinadas as seguintes palavras: Sistema Único de Saúde/SUS com reabilitação/políticas públicas/história; e reabilitação com história ou assistência médica (separadamente). Em inglês foram utilizadas as palavras rehabilitation e public health, com o objetivo de localizar artigos nacionais que tivessem sido publicados em outra língua, ou que fossem informativos para noções históricas da reabilitação. Consultaram-se ainda leis federais e manuais do Ministério da Saúde. Esse material foi buscado junto à Coordenação de Reabilitação do Rio de Janeiro, na biblioteca da Fundação Instituto Oswaldo Cruz (FIOCRUZ) e na base de dados BIREME. Foram excluídos artigos que não contemplavam o tema ou aos quais os autores não obtiveram acesso.

\section{RESULTADOS}

Foram incluídos quatro livros $(3,8-$ 10), três manuais do Ministério da Saúde $(5,7,11)$, quatro artigos nacionais (1, 12-14), uma dissertação de mestrado (15) e uma tese de doutorado (16). Esses materiais foram revisados com foco na história do direito à saúde e no tratamento da reabilitação, conforme descrito a seguir.

\section{História das políticas de saúde}

A história das políticas de saúde no Brasil está inserida no contexto da história do Estado brasileiro, com início no período colonial, e do interesse em manter saudável a mão-de-obra, com grandes mudanças após a industrialização e a criação de fundos de aposentadoria e pensões (3). A partir do século XIX, a assistência à saúde começou a ter maior influência sobre as práticas populares, com a regulamentação do ensino e da prática médica e a criação de hospitais públicos para atender doenças que exigiam maior controle do Estado, como as doenças mentais, a hanseníase e a tuberculose $(3,8)$.

Na década de 1920, novas ações para o controle das doenças foram implementadas tanto na área da saúde pública quanto da assistência médica individual (17). Logo, foram instituídas as Caixas de Aposentadoria e Pensões (CAPs), uma espécie de seguro social para algumas organizações trabalhistas ligadas à produção exportadora $(12,18)$. $\mathrm{O}$ direito à assistência e à saúde era restrito aos segurados (3).

Somente na década de 1930, com o crescimento da industrialização, instituiu-se um sistema de proteção social, com a criação dos Institutos de Aposentadorias e Pensões (IAPs), que ampliaram o papel das CAPs (3). Contudo, os IAPs ainda mantinham a exigência de contribuição trabalhista para a garantia do benefício, que era discriminado de acordo com a categoria profissional (3, 12). Além disso, o Estado ainda mantinha ações de saúde pública de caráter coletivo para controle e prevenção de doenças transmissíveis e assistência a algumas doenças (12).

Na década de 1950, o hospital tornouse o principal ponto de referência para a busca de atendimentos de saúde. $\mathrm{O}$ modelo de saúde manteve a sua organização em dois grupos: ações e serviços de saúde pública e sistema previdenciário, com políticas isoladas que atendiam a diferentes objetivos (3). Também nessa época foi criado o Ministério da Saúde, que se dedicava às atividades coletivas de assistência à saúde (12).

Em 1966, foi criado o INPS, unificando os IAP e centralizando a previdência social. A consequência mais evidente da concentração do poder dos IAPs foi a uniformização dos benefícios prestados à população atendida. Nesse período, a previdência social se firmou como principal órgão de financiamento dos serviços de saúde, com extensão da cobertura assistencial $(3,12,13)$.

A política proposta pelo INPS incluiu, no início da década de 1970, novas categorias profissionais, mas permanecia a exigência de comprovação do vínculo com o INPS através de carteira de trabalho ou carnê de contribuição para garantir a assistência hospitalar. Assim, muitos cidadãos continuavam sem direito à atenção à saúde (3). Nessa década, o Ministério da Saúde manteve suas ações de vigilância sanitária e epidemiológica, de vacinação e os programas contra determinadas doenças, enquanto a assistência médica individual ficava centrada no INPS. A política assistencial privilegiou a privatização dos serviços $\mathrm{e}$ estimulou o desenvolvimento de atividades hospitalares financiadas pelo Estado $(12,13)$.

Ainda na década de 1970, tentou-se regulamentar o papel do município na política de saúde através da lei 6 229/75 (12). De acordo com essa lei, os municípios deveriam manter e avaliar os serviços assistenciais e as ações de controle epidemiológico, bem como articular e integrar os serviços do sistema nacional de saúde. Contudo, a municipalização não prosperou, sendo dada pouca atenção aos atendimentos primários e à expansão da cobertura. De fato, não havia um sistema; as ações eram desenvolvidas de maneira fragmentada e sem integração. Ao Ministério da Saúde cabia a responsabilidade da formulação de políticas e ações de caráter coletivo, e ao Ministério da Previdência e Assistência So- 
cial a realização da assistência médica através dos Instituto Nacional de Assistência Médica e Previdência Social (INAMPS), que efetivamente controlava e avaliava as ações de saúde. A participação dos municípios era marginal e limitada ao repasse de dados quantitativos dessa produção assistencial $(9,12)$.

O volume cada vez maior de recursos exigido para a medicina curativa iniciou uma crise no sistema previdenciário e apontou para a necessidade de repensar a política de saúde $(3,19)$. Com um cenário de crise política, institucional e econômica decorrente do regime militar e da composição de uma política de desenvolvimento que tinha como meta básica o desenvolvimento social (entendido como desenvolvimento de políticas públicas de saúde, saneamento e habitação), houve um fortalecimento do movimento sanitário (3), que buscava reverter a lógica da assistência à saúde no Brasil, pregando a saúde como direito de todo o cidadão, a integração das ações de saúde em um único sistema, a gestão administrativa descentralizada para estados e municípios e a participação e controle social das ações de saúde pelo Estado.

As pressões por reformas na política de saúde possibilitaram algumas mudanças concretas ainda nos anos 1970, como a criação do INAMPS, mas ainda de forma incipiente e de acordo com os interesses do Estado. Entretanto, essas medidas favoreceram a construção de uma política de saúde mais universal, com prioridade para a extensão da oferta de serviços básicos $(3,7)$.

Nos anos 1980, o movimento da reforma sanitária na área da saúde indicava propostas de expansão de assistência médica na previdência social. Esse movimento criticava a mercantilização da medicina sob o comando da previdência social e buscava a universalização do direito à saúde, ampliando esse debate no Brasil. A partir disso, medidas de reformulação do sistema de saúde foram encaminhadas ao Estado, com base em um diagnóstico do modelo de saúde vigente que revelou uma rede de saúde ineficiente, desintegrada e complexa $(3,13)$.

Nesse cenário, ocorreu, em 1986, a VIII Conferência Nacional de Saúde, com a participação da comunidade e dos técnicos na discussão de uma política setorial. Nessa conferência foi aprovada a diretriz da universalização da saúde, sendo constituído o Sistema Unificado e Descentralizado da Saúde (SUDS), que se apresentou como estratégia-ponte para a construção do SUS $(3,12,13)$.

O SUS foi finalmente aprovado na Assembleia Nacional Constituinte de 1987/88, sendo suas diretrizes estabelecidas e definidas na Constituição de 1988. Essa Constituição Federal deu nova forma à saúde no Brasil, estabelecendo-a como direito universal e concebendo-a de maneira integral, preventiva e curativa $(3,7,12)$.

O SUS rompeu definitivamente com o padrão político anterior e afirmou um compromisso de proteção social abrangente, justo e democrático, onde o Estado tem o dever de promover a atenção à saúde mediante políticas sociais e econômicas, que garantam o acesso universal e igualitário às ações e serviços para promoção, proteção e recuperação da saúde $(3,7,20)$. Ocorreu, portanto, a descentralização do poder decisório do governo federal para os vários níveis de governo (União, estados e municípios), sendo as responsabilidades redistribuídas quanto às ações e aos serviços de saúde $(3,13)$.

Após a legalização do SUS, novas leis e portarias foram criadas pelo Ministério da Saúde a fim de garantir o financiamento e a regularização de diversos pontos dessa política, como a participação do setor privado. No entanto, ainda existe um longo caminho a ser trilhado $(3,12)$. Embora muitos municípios tenham avançado na garantia do direito universal e integral à saúde, essa não é a realidade de todo o país; alguns municípios encontram dificuldades na implementação das propostas, gerando sérios prejuízos à saúde da população (3). É justamente nesse contexto que se insere a atenção à saúde das pessoas com necessidades especiais.

\section{O caminho da reabilitação no Brasil}

Em conformidade com os princípios democráticos de saúde instituídos pela constituição de 1988, os direitos das pessoas com deficiência estão assegurados nos mais diferentes campos e aspectos (7). Nessa perspectiva da atenção integral à saúde, o Ministério da Saúde tem tentado viabilizar, através de uma série de atos legais, a inclusão da atenção à saúde da população com necessidades especiais, com um modelo assistencial pautado por abordagem multiprofissional e multidisciplinar, com ênfase nas ações de promoção à saúde, na reabilitação e na inclusão social (5).
A utilização de recursos de reabilitação na assistência à saúde teve início mundialmente no século XIX, com o advento da industrialização e os inúmeros acidentes de trabalho. Porém, os serviços de reabilitação passaram a ter importância somente no início do século XX, com o tratamento de sequelados do pósguerra e da epidemia de poliomielite nos Estados Unidos $(10,21)$. Nesse período, as leis de proteção à saúde conquistadas pelos trabalhadores, juntamente com a necessidade de recuperar a força de trabalho dos sequelados da guerra, foram importantes marcos para a expansão da reabilitação em todo o mundo (16).

Nas décadas de 1940 e 1950, com a sobrevivência de um maior número de crianças portadoras de distúrbios neurológicos e retardo mental e o consequente surgimento de técnicas de tratamento, houve uma preocupação com a possibilidade de reabilitação desses indivíduos $(16,22)$. Assim, nos anos 1960, surgiram os programas de estimulação precoce para crianças, especialmente nos Estados Unidos, com base em três premissas: a convicção da responsabilidade da sociedade de cuidar e proteger crianças e lactentes; o compromisso com as necessidades especiais das crianças que são vulneráveis às disfunções neuropsicomotoras; e o senso de que prevenir é melhor do que tratar e de que a intervenção precoce é melhor do que a remediação tardia (23).

Embora o Brasil tenha acompanhado esses programas de reabilitação, a assistência ao portador de necessidades especiais não era de competência de nenhuma esfera governamental, à exceção de algumas poucas instituições públicas (2). As primeiras instituições brasileiras criadas para portadores de deficiências físicas surgiram nos anos 1940, por iniciativa da sociedade civil (16). Os serviços ficavam a cargo de instituições filantrópicas e de caridade ou de atendimentos particulares e eram voltados principalmente à criança e particularmente aos portadores de deficiência mental $(1,2,5,24)$. Ainda nessa década, foram criadas leis contemplando a assistência a trabalhadores incapacitados que lançavam sobre os IAPs a responsabilidade de criar serviços dessa natureza, o que ocorreu somente em dois IAPs, o dos industriários e o dos comerciários (16).

No Brasil, até os anos 1960, as políticas de atenção à pessoa com necessidades especiais tiveram como paradigma prin- 
cipal a institucionalização e, consequentemente, a segregação. A institucionalização deveria ser de caráter transitório; no entanto, isso não era possível devido à precariedade dos serviços de reabilitação e à inconsistência do trabalho psicossocial com as famílias para viabilizar a reintegração do indivíduo na sociedade, permanecendo o mesmo excluído $(1,5)$.

Ainda na década de 1960, a reabilitação era compreendida como nível terciário da assistência e as únicas instituições públicas eram os centros de reabilitação profissional do INPS (6). Houve, porém, um aumento no número de instituições especializadas privadas (16). As políticas públicas na época eram incapazes de fazer a correta identificação e avaliação dos problemas pertinentes às pessoas com deficiência. Dessa forma, não havia políticas sociais que incluíssem a habilitação/reabilitação de pessoas com necessidades especiais (1).

A partir da década de 1970 ocorreu a desinstitucionalização de indivíduos incapacitados ou com retardo mental (21). Houve uma preocupação com a socialização do deficiente a partir da Declaração dos Direitos do Deficiente Mental da Organização das Nações Unidas (ONU), de 1971, que estabeleceu direitos para pessoas com necessidades especiais, incluindo o direito a atenção médica e reabilitação, assim como o direito de retornar ao cuidado familiar e de ser inserido produtivamente na sociedade (25).

No Brasil, a previdência social oferecia serviços de reabilitação aos trabalhadores acidentados por meio de 14 Centros de Reabilitação Profissional existentes no país. Ao mesmo tempo, as pessoas com necessidades especiais eram assistidas de maneira instável por serviços de reabilitação em hospitais, escolas especiais e instituições privadas que estabeleceram convênio com organizações como a Legião Brasileira de Assistência (LBA), a Fundação Nacional para o Bem Estar do Menor (FUNABEM) e o INPS (15).

Na década de 1980, marcada pela criação do SUS, os programas de reabilitação passaram a ter administração federal e consequente organização centralizada, passando os atendimentos em reabilitação a estar disponíveis para deficientes na rede pública de saúde (2). A participação das organizações ligadas aos portadores de deficiências na construção do SUS foi de extrema importância política e histórica, uma vez que en- fatizou o delineamento de uma organização dos serviços de reabilitação (15). No entanto, os serviços de reabilitação foram construídos de forma privilegiadamente urbana, concentrados nas regiões economicamente mais favorecidas, com cobertura assistencial baixa e organizados por tipo de deficiência $(2,15)$.

Com o repasse dos serviços de saúde aos municípios, as secretarias municipais de saúde assumiram o desafio de oferecer de maneira mais sistemática as ações voltadas para o atendimento integral às múltiplas necessidades do deficiente (1). Entretanto, ainda na década de 1980, os programas de reabilitação tinham, na maioria dos casos, uma postura assistencialista, ao invés de uma abordagem integradora. Contudo, devese lembrar que foram propostas pioneiras para esse segmento populacional (5).

No final da década de 1980, foi promulgada a lei $7853 / 89$, que propunha o apoio às pessoas portadoras de deficiência e a sua integração social. No que se refere ao setor saúde, essa lei atribuiu ao setor, dentre outros aspectos, a responsabilidade pela criação de uma rede de serviços especializados em reabilitação e habilitação (5). Iniciou-se assim, ainda que timidamente, a organização das redes municipais de reabilitação. Segundo Bertoti (26), a década de 1990 foi mundialmente conhecida como a era do "assistencialismo", uma vez que foi iniciada a oferta da assistência necessária para maximizar a integração do indivíduo. Contudo, apesar dos esforços dos estados em implantar serviços públicos de reabilitação, ainda boa parte da assistência ocorreu por meio de instituições filantrópicas financiadas por recursos públicos (16). Em 1993, com o Programa de Atenção à Saúde da Pessoa Portadora de Deficiência, do Ministério da Saúde, o SUS passou a incentivar a criação de centros de reabilitação multiprofissionais especializados como forma de acompanhar e estimular o desenvolvimento de indivíduos com déficits motores, sensoriais ou cognitivos (2).

Com base no conhecimento adquirido no século XX, o século XXI começou com grandes expectativas em relação aos programas de estimulação precoce para a geração de inclusão social (23). No ano de 2001, as redes estaduais de assistência à pessoa portadora de deficiência passaram a ser organizadas por nível de complexidade (primário, intermediário e alta complexidade) (27). Coube, assim, a cada secretaria estadual de saúde determinar a necessidade de sua cobertura assistencial.

Em 2004, o governo federal instituiu a Agenda de Compromisso para Saúde Integral da Criança e Redução da Mortalidade Infantil. Esse documento, que apresenta as principais diretrizes a serem seguidas para o cuidado integral da criança, destacou a prevenção de possíveis deficiências psicomotoras e a atenção a crianças com necessidades especiais. Foi enfatizada a importância da intervenção precoce multiprofissional para a reabilitação da criança em toda a sua capacidade funcional e, consequentemente, a promoção da qualidade de vida (28). No final do ano de 2004, entrou em vigor a portaria 2 607, que aprovava o Plano Nacional de Saúde (PNS), no qual foram apresentadas metas para a consolidação da rede de atenção à saúde da pessoa portadora de deficiência. A principal linha de atuação foi a concretização das redes estaduais de reabilitação, visando a potencializar a capacidade funcional do indivíduo e a sua inclusão social (11).

Paralelamente à rede pública de reabilitação, tem-se a participação de instituições privadas, principalmente das instituições sem fins lucrativos, que recebem subsídio financeiro do Sistema Único de Assistência Social (SUAS). O SUAS foi criado com o objetivo de regularizar e organizar, em todo o território nacional, serviços, programas e benefícios socioassistenciais, em articulação com a sociedade civil. Nesse sistema, a linha de ação definida como proteção social básica engloba a atenção a pessoas com necessidades especiais, realizada através de ações socioeducacionais e, por consequência, de reabilitação, em instituições não-governamentais (29). O trabalho conjunto de instituições governamentais e filantrópicas deveria evitar a superposição ou a ausência de serviços, otimizar os recursos e gerar resultados mais significativos, uma vez que o número de pessoas com necessidades especiais é muito elevado e o Estado ainda não oferece serviços públicos suficientes a todas as regiões populacionais (14).

Atualmente, as ações de reabilitação no Brasil ainda se dão em uma rede precária e desarticulada, com dificuldade de comunicação, e não contemplam uma política integral de atendimento às incapacidades $(1,15)$. Há uma descontinuidade entre as ações das esferas pública e 
privada, sendo a assistência prestada a um número reduzido de pessoas (5). Contudo, as críticas a essa política de saúde devem ser atenuadas, pois ela se mostra pioneira na área de assistência a esse segmento da população (7).

\section{DISCUSSÃO}

A despeito da evolução das políticas públicas no Brasil e da instituição da saúde como direito de todos, o Estado ainda precisa implementar medidas que garantam ou suportem essa premissa. Entretanto, não é simples colocar em prática uma política tão abrangente. A reforma do sistema de saúde brasileiro ainda está em curso e só será finalizada se o Estado e a sociedade concordarem que há necessidade urgente de uma política mais justa, solidária e melhor distribuída no Brasil (4).

Apesar de todas as medidas tomadas até o momento, ainda persistem fatores que dificultam o alcance de ótimos resultados na atenção à pessoa com necessidades especiais, dentre os quais se destacam a desinformação da sociedade, a precária distribuição dos recursos financeiros e a visão limitada dos serviços sobre como podem contribuir para melhoria da qualidade de vida (5). Logo, cabe especialmente aos municípios o desafio de assumir o planejamento das ações, estabelecendo a oferta adequada de serviços e promovendo, assim, a equi- dade ao acesso e a integralidade da assistência (13).

Um exemplo é o Estado do Rio de Janeiro, que, segundo Almeida (15), é um dos que mais alcançou a descentralização dos serviços e ações de saúde e que já organiza os serviços de reabilitação de acordo com a normativa nacional. Em especial, o Município do Rio de Janeiro criou, em 2002, a Coordenação de Programas de Reabilitação, com o objetivo de articular as diversas especialidades terapêuticas e iniciar a implantação de uma rede de reabilitação hierarquizada por níveis de complexidade, de modo a assegurar ao usuário uma rede de referência e contrarreferência, além de tentar contemplar as necessidades de cada área programática, garantindo melhor acessibilidade e integralidade de assistência (1). Contudo, essa rede, que ainda se encontra em construção, permanece desarticulada de outros setores da prefeitura: por exemplo, não está disponível à sociedade e à comunidade médica uma listagem oficial com todos os locais de atendimento (públicos e privados) do Município do Rio de Janeiro.

A articulação entre as esferas governamentais e as ações não-governamentais mostra-se imprescindível, sobretudo para apontar necessidades, sugerir soluções ou oferecer serviços complementares às pessoas portadoras de deficiência (5). Entretanto, tal articulação e parceria encontram-se ainda em pro- cesso de construção, sendo permeadas por disputas de interesses (13).

Diante disso, faz-se necessária a criação de uma rede de reabilitação articulada com atribuições específicas para cada serviço, público e/ou privado, evitando a superposição. Outrossim, é imprescindível a formalização de um sistema de informação acessível à sociedade e à comunidade médica sobre os locais disponíveis de atendimento, bem como o tipo de assistência oferecida, de forma a tornar eficiente o sistema de referência e contrarreferência.

Além das importantes lacunas no desenho da proposta do SUS, como o financiamento e descentralização das ações, outro aspecto importante é o fato de que os serviços, os profissionais de saúde e também a população aprenderam durante anos uma prática de saúde que não buscava o olhar integral. Com a reforma ocorrida no sistema de saúde é necessário incorporar e construir uma nova concepção de saúde, capaz de compreender o indivíduo em meio a uma coletividade (3).

Desse modo, sugere-se que o governo apoie pesquisas que busquem o diagnóstico da situação atual dos serviços de reabilitação em cada região administrativa. Também é preciso incentivar uma formação mais abrangente dos profissionais da área de saúde, que os habilite a compreender o indivíduo em todas as suas necessidades.

\section{REFERÊNCIAS}

1. Coelho AEBD, Lobo ST. Gestão participativa na organização de uma rede de reabilitação em saúde pública. Rev Virt Gestão Iniciat Soc. 2004;1(out)37-45.

2. Brasil, Ministério da Saúde, Coordenação de Atenção a Grupos Especiais. Programa de Atenção à Saúde da pessoa portadora de deficiência. Atenção à pessoa portadora de deficiência no Sistema Único de Saúde: planejamento e organização de serviços. Brasília: Secretaria de Assistência à Saúde; 1993. Disponível em: www.inep.gov.br/PES QUISA/BBE-ONLINE/det.asp? $\operatorname{cod}=53152$ \&type $=$ M. Acessado em 26 de novembro de 2009.

3. Baptista TWF. O direito à saúde no Brasil: sobre como chegamos ao sistema único de saúde e o que esperamos dele. Rio de Janeiro: Fiocruz; 2005. Pp. 11-41. [Fundação Oswaldo Cruz, Escola Politécnica de Saúde Joaquim Venâncio. Textos de apoio em políticas de saúde].
4. Brasil, Presidência da República. Lei 7 853, de 24 de outubro de 1989. Disponível em: www.planalto.gov.br/CCIVIL/LEIS/L7853. $\mathrm{htm}$. Acessado em 26 de novembro de 2009.

5. Brasil, Ministério da Saúde, Secretaria de Atenção à Saúde. Manual de legislação em saúde da pessoa com deficiência. Brasília: Ministério da Saúde; 2006. Disponível em: bvsms.saude.gov.br/bvs/publicacoes/legislacao_deficiencia.pdf. Acessado em 26 de novembro de 2009.

6. Brasil, Ministério da Saúde, Secretaria de Assistência à Saúde, Coordenação de atenção à saúde da pessoa portadora de deficiência. Atenção à pessoa portadora de deficiência no Sistema Único de Saúde: planejamento e organização de serviços. Brasília: Ministério da Saúde; 1995.

7. Faleiros VP, Silva JFS, Vasconcellos LCF, Silveira RMG. A construção do SUS: história da reforma sanitária e do processo participativo. Brasília: Ministério da Saúde; 2006.
Disponível em: portal.saude.gov.br/portal/ arquivos/pdf/construcao_do_SUS.pdf. Acessado em dezembro de 2009.

8. Costa JF. História da psiquiatria no Brasil: um corte ideológico. Rio de Janeiro: Xenon: 1989.

9. Santos L. Distribuição de competências no Sistema Único de Saúde: o papel do Estado nas três esferas de governo no SUS. Brasília: Organização Pan-Americana da Saúde/Organização Mundial da Saúde; 1994.

10. Rebelatto JR, Botome SP. Fisioterapia no Brasil: fundamentos para uma ação preventiva e perspectivas profissionais. $2^{\mathrm{a}}$ ed. São Paulo: Manole; 1999.

11. Brasil, Ministério da Saúde, Secretaria Executiva, Subsecretaria de Planejamento e Orçamento. Plano Nacional de Saúde: um pacto pela saúde do Brasil. Brasília: Ministério da Saúde; 2005. Disponível em: dtr2001.saude. gov.br/editora/produtos/livros/pdf/05_03 06_M.pdf. Acessado em novembro de 2009. 
12. Paulus Jr A, Cordoni Jr L. Políticas públicas de saúde no Brasil. Rev Espac Saude. 2006; 8(1):13-9.

13. Santos FP, Merhy EE. A regulação pública da saúde no Estado brasileiro: uma revisão. Interface Comun Saude Educ. 2006;10(19): 25-41.

14. Cruz G, Peralta RL. CIAD: Uma experiência de política pública integrada. Rev Virt Gestão Iniciat Soc. 2004;1(out):3-6.

15. Almeida LGR. Estudos sobre a distribuição dos serviços de reabilitação: o caso do Rio de Janeiro [dissertação]. Rio de Janeiro: Escola Nacional de Saúde Pública; 2004

16. Almeida MC. Saúde e reabilitação de pessoas com deficiência: políticas e modelos assistenciais [tese]. Campinas: Universidade Estadual de Campinas. 2000.

17. Costa NR. Lutas urbanas e controle sanitário: origens das políticas de saúde no Brasil. Rio de janeiro: Vozes; 1985.

18. Oliveira JA, Teixeira SM. (Im)Previdência Social: 60 anos de história da previdência no Brasil. Rio de Janeiro: Vozes; 1985.
19. Braga JCS, Paula SG. Saúde e previdência: estudos de política social. São Paulo: Hucitec; 1986.

20. Brasil, Presidência da República. Constituição Federal do Brasil de 1988. Artigo 196. Disponível em: www.dji.com.br/constitu icao_federal/cf196a200.htm. Acessado em 26 de novembro de 2009.

21. Ratliffe KT. Fisioterapia na clínica pediátrica. São Paulo: Santos; 2002.

22. Flehming I. Texto e atlas do desenvolvimento motor normal e seus desvios no lactente: diagnóstico e tratamento precoce do nascimento até o $18^{\circ}$ mês. São Paulo: Atheneu; 2002.

23. Meisels SJ, Shonkoff JPE. Early childhood intervention: a continuing evolution. Em: Shonkoff JP, Meisels SJ, eds. Handbook of early childhood intervention. $2^{\mathrm{a}}$ ed. Nova Iorque: Cambridge University; 2000. Pp. 3-31.

24. Silva OM. Políticas para pessoas portadoras de deficiência: "o papel e a influência das organizações internacionais". Mensagem da APAE. 1992;65:22-6.
25. Vital J. A proteção do excepcional na Previdência Social. Mensagem da APAE. 1975;2: 79-80.

26. Bertoti DB. Retardo mental: foco na síndrome de Down. Em: Tecklin JS, ed. Fisioterapia pediátrica. 3a ed. Porto Alegre: Artmed; 2002. Pp. 236-56.

27. Brasil, Ministério da Saúde. Portaria $\mathrm{n}^{\circ}$ 818/GM em 05 de junho de 2001. Disponível em: dtr2001.saude.gov.br/sas/PORTARIAS/ Port2001/GM/GM-818.htm. Acessado em 26 de novembro de 2009.

28. Brasil, Ministério da Saúde, Secretaria de Atenção à Saúde. Agenda de compromisso para a saúde integral e redução da mortalidade infantil. Brasília: Ministério da Saúde; 2004

29. Sistema Único de Assistência social (SUAS) [site da Internet]. Disponível em: www.mds. gov.br/programas/rede-suas. Acessado em dezembro de 2009.

Manuscrito recebido em 15 de junho de 2009. Aceito em versão revisada em 7 de agosto de 2009.

ABSTRACT Objective. To produce a historical account of rehabilitation actions in the context of the Brazilian Unified Health Care System (SUS).

Methods. Search of SciELO, LILACS, and MEDLINE databases for literature pub-

The public health care system and rehabilitation actions in Brazil

Key words lished between 1980 and 2009. The following Portuguese search terms were used: Sistema Único de Saúde, reabilitação, politicas de saúde, assistência médica, história. The English terms "rehabilitation" and "public health" were also used. Federal laws and Ministry of Health manuals available at the city of Rio de Janeiro Coordinating Office for Rehabilitation Programs, Fundação Instituto Oswaldo Cruz library, and in BIREME database were also surveyed.

Results. Only a small number of publications were recovered (four books, three Health Ministry manuals, four articles published in Brazil, one master's thesis, and one doctoral dissertation). Nevertheless, analysis of these materials revealed that since many municipalities are still incapable of ensuring the right to universal and comprehensive health care, rehabilitation actions are often carried out in a precarious manner, unsupported by an adequate and comprehensive policy. On the other hand, there have been real improvements in terms of expanding care to the population with special needs.

Conclusions. There still are factors hindering the achievement of optimal results in the care to people with special needs. The challenge of action planning must be undertaken especially by municipal governments to ensure an adequate supply of services and thus equity of access and comprehensive health care.

Public policies; rehabilitation; Single Health System; Brazil. 